\title{
First-Line Sofia Aspiration Thrombectomy Approach within the Endovascular Treatment of Ischemic Stroke Multicentric Registry: Efficacy, Safety, and Predictive Factors of Success
}

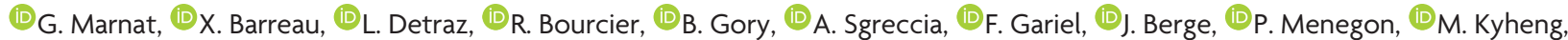 \\ (D). Labreuche, (D)A. Consoli, DR. Blanc, and DB. Lapergue, on behalf of the ETIS Investigators
}

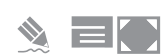

\begin{abstract}
BACKGROUND AND PURPOSE: After publications on the effectiveness of mechanical thrombectomy by stent retrievers in acute ischemic stroke with large-vessel occlusion, alternative endovascular approaches have been proposed using first-line aspiration catheters. Several devices are currently available to perform A Direct Aspiration First Pass Technique. The Sofia catheter aspiration has been widely used by interventionalists, but data are scarce about its efficacy and safety. Our aim was to report our multicenter thrombectomy experience with first-line Sofia catheter aspiration and to identify independent prognostic factors of clinical and procedural outcomes.
\end{abstract}

MATERIALS AND METHODS: We performed a retrospective analysis of the prospectively maintained Endovascular Treatment of Ischemic Stroke multicentric registry. Data from consecutive patients who benefited from thrombectomy with a first-line Sofia approach between January 2013 and April 2018 were studied. We excluded other first-line approaches (stent retriever or combined aspiration and stent retriever) and extracranial occlusions. Baseline characteristics, procedural data, and angiographic and clinical outcomes were analyzed.

RESULTS: During the study period, 296 patients were treated. Mean age and initial NIHSS score were, respectively, 69.5 years and 16. Successful reperfusion, defined by the modified $\mathrm{TICl} 2 \mathrm{~b} / 3$, was obtained in $86.1 \%(n=255 ; 95 \% \mathrm{Cl}$, 81.7\%-89.9\%). Complete reperfusion (modified TICl 3 ) was obtained in $41.2 \%(n=122 ; 95 \% \mathrm{Cl}, 35.5 \%-47.1 \%)$. A first-pass effect was achieved in $24.2 \%(n=71 ; 95 \% \mathrm{Cl}, 19.4 \%-29.6 \%)$. A rescue stent retriever approach was required in $29.7 \%(n=88 ; 95 \% \mathrm{Cl}, 24.6 \%-35.3 \%)$. The complication rate was $9.5 \%(n=28 ; 95 \% \mathrm{Cl}, 6.4 \%-13.5 \%)$. Forty-three percent ( $n=122 ; 95 \% \mathrm{Cl}, 37.1 \%-48.9 \%$ ) of patients presented with a favorable 3-month outcome (mRS $\leq 2)$. Older age, M1-occlusion topography, and intravenous thrombolysis use prior to thrombectomy were independent predictors of the first-pass effect.

CONCLUSIONS: The first-line contact aspiration approach appeared safe and efficient with Sofia catheters. These devices achieved very high reperfusion rates with a low requirement for stent retriever rescue therapy, especially for $\mathrm{Ml}$ occlusions.

ABBREVIATIONS: ADAPT $=$ A Direct Aspiration First Pass Technique; DAC $=$ distal aspiration catheter; $\mathrm{ETIS}=$ Endovascular Treatment of Ischemic Stroke; FPE $=$ first-pass effect; IQR = interquartile range; IVT = intravenous thrombolysis; $\mathrm{mTICl}=$ modified $\mathrm{TICl} ; \mathrm{PH}$ = parenchymal hematoma

A fter publication of the major trials reporting the efficacy of mechanical thrombectomy for large-vessel occlusion strokes, ${ }^{1}$ the principal concern is now to optimize recanalization rates. Recent advances in thrombectomy devices progressively enabled major improvement. In addition to stent retrievers, contact aspi-

Received January 9, 2019; accepted after revision April 18.

From the Interventional and Diagnostic Neuroradiology Department (G.M., X.B., F.G., J.B., P.M.), Bordeaux University Hospital, Bordeaux, France; Interventional and Diagnostic Neuroradiology Department (L.D., R.B.), Nantes University Hospital, Nantes, France; Department of Diagnostic and Therapeutic Neuroradiology (B.G.), University Hospital of Nancy, Nancy, France; Institut National de la Santé et de la Recherche Médicale U1254 (B.G.), IADI F-54000, Nancy, France; Department of Radiology (A.S.), Ospedali Riuniti, Siena, Italy; Université Lille (M.K., J.L.), Centre Hospitalier Universitaire Lille, Lille, France; Department of Neuroradiology (A.C.), Foch Hospital, Suresnes, France; Interventional Neuroradiology Department (R.B.), Fondation Ophtalmologique Rothschild, Paris, France; and Department of Neurology (B.L.), Stroke Center, Foch Hospital, Suresnes, France; ETIS Collaborators are in the On-line Appendix.

Financial support was provided by MicroVention (Tustin, California). ration has greatly modified the endovascular approach and is now considered a routine technique. Since the A Direct Aspiration First Pass Technique (ADAPT) was described, ${ }^{2,3}$ various distal aspiration catheter (DAC) technologies have been developed. ADAPT consists of contact thrombus suction through a largediameter catheter. This is a seductive first-line approach because it appears effective, safe, fast, and cost-effective. ${ }^{4}$ With recent publications of the Contact Aspiration Versus Stent Retriever for

\footnotetext{
The funders had no role in the design and conduct of the study; collection, management, analysis, and interpretation of the data; preparation, review, or approval of the manuscript; or decision to submit the manuscript for publication.

Please address correspondence to Gaultier Marnat, MD, Interventional and Diagnostic Neuroradiology Department, Bordeaux University Hospital, Hôpital Pellegrin, Place Amélie Raba-Léon, 33000 Bordeaux, France; e-mail: gaultier.marnat@chu-bordeaux.fr

E Indicates article with supplemental on-line appendix and tables.

Indicates article with supplemental on-line photo.

http://dx.doi.org/10.3174/ajnr.A6074
} 
Successful Revascularization (ASTER) and Aspiration thrombectomy versus stent retriever thrombectomy as first-line approach for large vessel occlusion (COMPASS) trials, ADAPT can now be considered a first-line option in the thrombectomy strategy. ${ }^{5,6}$ In case of aspiration failure, the endovascular technique can then be converted to a combined aspiration + stent retriever approach. ${ }^{7}$ These DACs must have specific characteristics such as navigability, torqueability, visibility, kink resistance, and aspiration capacity to obtain higher performance levels.

Sofia (soft torqueable catheter optimized for intracranial access; MicroVention, Tustin, California) is a DAC with a specific hybrid design. Its braid and coil construction combines different softness segments with a distal inner lumen of 0.055-0.070 inches, respectively, in $5 \mathrm{~F}$ and $6 \mathrm{~F}$ Plus versions. Despite previous publications, ${ }^{8-11}$ the safety and efficacy of this DAC in a large population with first-line Sofia use are yet to be reported.

Recanalization is a strong predictor of favorable outcome. Especially, the recently described first-pass effect (FPE), defined as a complete recanalization after 1 pass of mechanical thrombectomy, has been related to substantial improvement in clinical outcome compared with final good recanalization results after numerous passes. ${ }^{12}$ However, in the North American Solitaire Stent-Retriever Acute Stroke registry study, ${ }^{12}$ most patients were treated with a first-line stent retriever strategy. Data regarding the FPE under the ADAPT strategy are still undereported to date.

We present here the largest multicentric experience to date of the first-line ADAPT strategy using Sofia $6 \mathrm{~F}$ Plus or 5F catheters. We aimed to study the clinical and angiographic effectiveness with specific attention to predictive factors of the first-pass effect.

\section{MATERIALS AND METHODS Study Design and Population}

We performed a retrospective analysis of our prospectively maintained, multicentric Endovascular Treatment of Ischemic Stroke (ETIS) data base. ETIS is a prospective registry collecting data from patients benefiting from mechanical thrombectomy in 4 major French comprehensive stroke centers. Local ethics committees approved this study.

Consecutive patients from January 2013 to April 2018, presenting with acute ischemic stroke due to large-vessel occlusion strokes of the anterior or posterior circulation treated by mechanical thrombectomy using first-line ADAPT with a Sofia catheter were analyzed. We excluded patients treated first-line by another DAC, stent retrievers combined with distal aspiration, or stent retrievers alone. Extracranial occlusions were also excluded.

\section{Treatment Protocol}

The indication for treatment was left to the discretion of each operator according to local protocol. Intravenous thrombolysis treatment (IVT) was administered in association with mechanical thrombectomy in patients treated within 4.5 hours of stroke onset in the absence of contraindications. Endovascular treatment was performed with the patient under local anesthesia alone, conscious sedation, or general anesthesia depending on the patient's condition and each contributing center's anesthesia protocol.

For all selected cases, the operator's decision was to perform distal aspiration through a Sofia catheter as the first intention.
Sofia catheter choice was made according to operator's discretion, local protocols, catheter availability, and occlusion topography to fit with the ADAPT strategy as a first-line treatment option. First, an $8 \mathrm{~F}$ or $9 \mathrm{~F}$ guide catheter was placed into the cervical internal carotid artery. Then, a Sofia $6 \mathrm{~F}$ Plus or $5 \mathrm{~F}$ was advanced over a microwire to the occlusion site with or without microcatheter support, depending on cervical and intracranial ICA tortuosity. The use of a balloon-guide catheter was left to the interventionist's discretion. A 3-minute aspiration period, using either a dedicated pump or manual depression through a vacuum syringe, was applied before DAC removal. In the case of failure or partial efficacy, this maneuver could be repeated. In case of ADAPT failure or insufficient recanalization, the choice to convert to another endovascular technique (distal aspiration combined with stent retrievers or stent retrievers alone) was left to the operator's discretion. Complementary endovascular treatments, such as cervical or intracranial angioplasty and/or stent placement, were performed depending on stroke etiology and severity and angiographically detected underlying lesions.

\section{Data Collection and Outcome Evaluation}

Age, sex, medical history, previous medication, stroke severity using the NIHSS score, stroke etiology, imaging technique, ASPECTS, IVT, and angiographic (arterial occlusion topography, degree of revascularization assessed by the modified TICI [mTICI] score) data were recorded.

Clinical outcome was assessed by the mRS 3 months after stroke. Favorable outcome was defined by an $\mathrm{mRS} \leq 2$. Angiographic reperfusion was rated using the mTICI score. Successful revascularization was defined by mTICI $\geq 2$ b. Near-complete and excellent reperfusion was defined, respectively, by mTICI of $2 \mathrm{c} / 3$ and 3 . The FPE was defined as an mTICI 3 after a single aspiration maneuver.

Safety criteria were also recorded, including procedural complications (dissection, embolism in a new territory, and intracranial arterial perforation), intracranial hemorrhage, and mortality. Procedural times were analyzed.

\section{Statistical Analysis}

Categoric variables were expressed as frequencies and percentages. Quantitative variables were expressed as means or medians (interquartile range, [IQR]) for non-normal distribution. The normality of distributions was assessed graphically and using the Shapiro-Wilk test.

Associations of baseline characteristics (patient and treatment characteristics) with outcomes (favorable outcome, successful and excellent reperfusion, FPE) were first investigated in centeradjusted analyses using generalized linear mixed models by including center as a random effect. To assess the independent predictors of each outcome, we entered all patient and treatment characteristics with a $P<.20$ in center-adjusted analyses into a backward-stepwise multivariable generalized linear mixed model using a removal criterion of $P>.05$. Before developing the multivariable prognostic models, we examined the log-linearity assumption for continuous characteristics using restricted cubic spline functions as well as the absence of collinearity between candidate predictors by calculating the variance inflation factors. To avoid case deletion in analyses due to missing data on baseline 


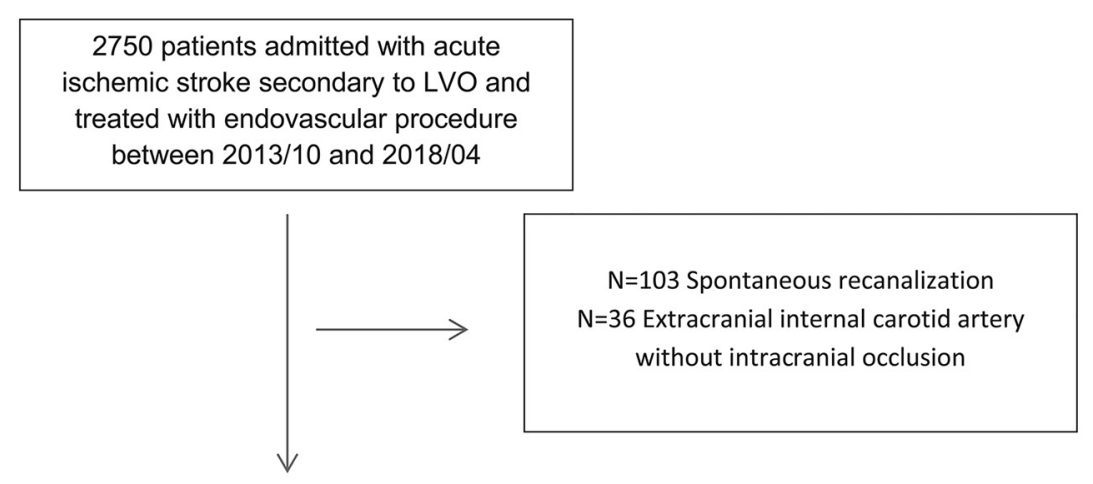

2611 AIS treated by EVT

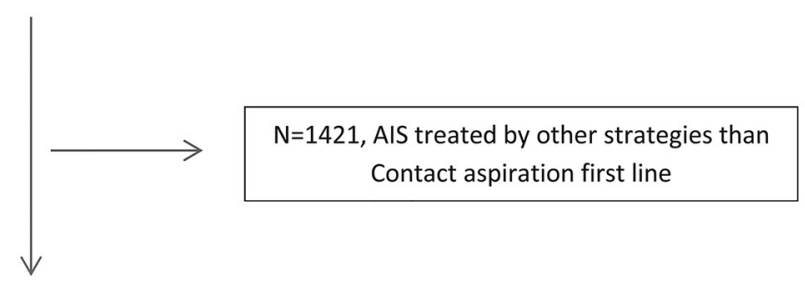

1190 AIS treated by first-line Contact Aspiration

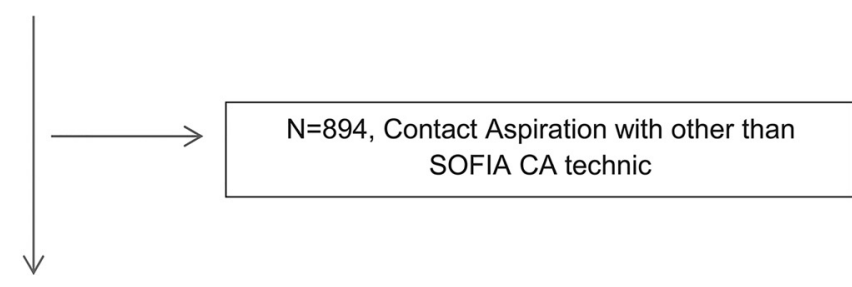

296 AIS treated by SOFIA aspiration contact catheter included in the primary analysis

FIGURE. Flowchart of the Sofia-Contact Aspiration, First-Line Technique Registry. AIS indicates acute ischemic stroke; LVO, large vessel occlusion; EVT, endovascular treatment; CA, contact aspiration.

characteristics and outcomes, we imputed missing data by multiple imputations using a regression-switching approach (chained equations with $\mathrm{m}=10$ ). The imputation procedure was performed under the missing at random assumption using all baseline characteristics and study outcomes with a predictive mean matching method for continuous variables and a multinomial or binary logistic regression model for categoric variables. Estimates obtained in the different imputed datasets were combined using the Rubin rules. Finally, we examined the performance of the final prognostic models in terms of discrimination by calculating the C-statistics in each imputed dataset and by reporting the median and range values.

Statistical testing was performed at the 2-tailed $\alpha$ level of .05 . Data were analyzed using the SAS software package, Release 9.4 (SAS Institute, Cary, North Carolina).

\section{RESULTS}

\section{Population and Participating Centers}

From October 2013 to April 2018, we treated 2750 patients with an arterial large-vessel occlusion by mechanical thrombectomy at
4 comprehensive stroke centers. Of these, 296 (10.8\%) who had undergone endovascular treatment with the Sofia aspiration catheter as a first-line approach were included in the present study (center A: 73 patients; B: 103 patients, C: 92 patients, D: 28 patients) (Figure).

The main patient and treatment characteristics are reported in Table 1. Overall, the mean age was $69.5 \pm 13.8$ years; $50.7 \%(n=150)$ were men, and the median admission NIHSS score was 16 (IQR, 11-21). Intravenous tPA before endovascular treatment was administered in $48.3 \%(n=142)$ of patients. The median time from symptom onset to arterial puncture was 242 minutes (IQR, 186-324 minutes). Most patients were treated with a $6 \mathrm{~F}$ Sofia catheter ( $n=226,76.3 \%$ ), and the remaining 70 $(23.7 \%)$ were treated with a $5 \mathrm{~F}$ catheter.

\section{Effectiveness and Safety Outcomes}

The main safety and effectiveness outcomes are summarized in Table 2. Thrombectomy was effective in $86.1 \%$ $(n=255$; 95\% CI, $81.7 \%-89.9 \%)$ of patients (successful reperfusion, mTICI 2b-3) with a median number of passes of 2 (IQR, 1-3) and a median time of 40 minutes (IQR, 24-65 minutes) from groin puncture. Excellent reperfusion (mTICI 3 ) was achieved in $41.2 \%$ of patients $(n=122 ; 95 \%$ CI, 35.5\%-47.1\%). The first-pass effect was achieved in $24.2 \%(n=71,95 \% \mathrm{CI}, 19.4-29.6 \%)$. Rescue therapy was required in $29.7 \%$ $(n=88$; 95\% CI, 24.6\%-35.3\%).

Procedural complications occurred in $9.5 \%(n=28$; $95 \% \mathrm{CI}, 6.4 \%-13.5 \%)$ of patients. These included $14(4.7 \%)$ new-territory embolisms, 4 (1.4\%) vessel perforations, 4 (1.4\%) extracranial carotid dissections, and 6 (2.0\%) vasospasms. Intracranial hemorrhage within 24 hours occurred in $44.6 \%$ ( $n=115$; 95\% CI, 38.4\%-50.9\%). Among these, there were $31(12.0 \%)$ parenchymal hematomas (PH-1 and $\mathrm{PH}-2)$ and 16 (6.2\%) symptomatic intracranial hemorrhages. At 90 days, $43 \%$ of patients $(n=122$; $95 \%$ CI, 37.1\%$48.9 \%$ ) were functionally independent (mRS $0-2$ ) and $22.9 \%$ had died ( $n=65 ; 95 \%$ CI, 18.1\%-28.2\%) (See the On-line Figure for the overall distribution of 90-day mRS). Results according to anterior or posterior occlusion topography are presented in On-line Tables 1 and 2.

Among patients treated with the ADAPT approach alone without the need for rescue therapy, successful reperfusion was obtained in 91.8\% ( $n=191$; 95\% CI, 87.2\%-95.2\%); 50.5\% ( $n=100$; 95\% CI, $43.3 \%-57.7 \%$ ) had a 90 -day favorable outcome. In this subgroup of patients, the mortality rate was $20.2 \%(n=40 ; 95 \%$ CI, $14.8 \%-$ 
Table 1: Baseline characteristics $(n=296)^{\mathrm{a}}$

\begin{tabular}{|c|c|c|}
\hline Characteristics & No. & Values \\
\hline \multicolumn{3}{|l|}{ Baseline demographics and medical history } \\
\hline Age (mean) (yr) & 296 & $69.5 \pm 13.8$ \\
\hline Men & 296 & $150(50.7)$ \\
\hline Hypertension & 288 & $158(54.9)$ \\
\hline Diabetes & 285 & $50(17.4)$ \\
\hline Dyslipidemia & 284 & $93(32.7)$ \\
\hline Current smoking & 272 & $70(25.7)$ \\
\hline Previous stroke or TIA & 286 & $43(15.0)$ \\
\hline Previous CAD & 285 & $50(17.5)$ \\
\hline Antithrombotic medications & 291 & $120(41.2)$ \\
\hline Antiplatelet & 291 & $69(23.7)$ \\
\hline Anticoagulant & 291 & 34 (11.7) \\
\hline \multicolumn{3}{|l|}{ Current stroke event } \\
\hline Systolic BP (mean) (mm Hg) & 245 & $148 \pm 28$ \\
\hline Diastolic BP (mean) (mm Hg) & 245 & $82 \pm 19$ \\
\hline Blood glucose (median) (IQR) (mmol/L) & 229 & $6.4(5.7-8.0)$ \\
\hline Admission NIHSS score (median) (IQR) & 290 & $16(11-21)$ \\
\hline ASPECTS (median) (IQR) & 278 & $7(6-9)$ \\
\hline Prestroke $\mathrm{mRS} \geq 1$ & 291 & $64(22.0)$ \\
\hline \multicolumn{3}{|l|}{ Site of occlusion } \\
\hline M1 MCA & 296 & $124(41.9)$ \\
\hline M2 MCA & & $40(13.5)$ \\
\hline T-car & & $54(18.2)$ \\
\hline Tandem & & 39 (13.2) \\
\hline Vertebrobasilar & & 39 (13.2) \\
\hline \multicolumn{3}{|l|}{ Stroke etiology } \\
\hline Large-artery atherosclerosis & 295 & $44(14.9)$ \\
\hline Cardioembolic & & $127(43.1)$ \\
\hline Dissection & & $11(3.7)$ \\
\hline Others & & $113(38.3)$ \\
\hline Intravenous rtPA & 294 & $142(48.3)$ \\
\hline General anesthesia & 291 & $71(24.4)$ \\
\hline \multicolumn{3}{|l|}{ Procedural times (median) (IQR) (min) } \\
\hline Onset to imaging & 279 & $113(84-180)$ \\
\hline Admission to imaging & 248 & $25(16-41.5)$ \\
\hline Imaging to groin puncture & 262 & 107 (69-151) \\
\hline Imaging to recanalization & 279 & $157(118-205)$ \\
\hline Onset to puncture & 276 & $242(186-324)$ \\
\hline Puncture to recanalization & 287 & $40(25-65.5)$ \\
\hline Balloon-guide catheter & 296 & $23(7.8)$ \\
\hline \multicolumn{3}{|l|}{ Mode of admission } \\
\hline Mothership & 295 & $121(41.0)$ \\
\hline Drip and ship & & $174(59.0)$ \\
\hline \multicolumn{3}{|l|}{ Sofia catheter } \\
\hline $5 \mathrm{~F}$ & 296 & 70 (23.7) \\
\hline $6 \mathrm{~F}$ & & $226(76.3)$ \\
\hline
\end{tabular}

Note:-BP indicates blood pressure; CAD, coronary artery disease; $\mathrm{T}$-car, occlusion of carotid terminus; Mothership, comprehensive stroke centers with on-site interventional neuroradiologic services; Drip and ship, medical treatment (including intravenous thrombolysis) in a hospital before being transferred to a comprehensive stroke centers for thrombectomy.

${ }^{a}$ Values are expressed as No. (percentage) unless otherwise indicated.

$26.5 \%)$ and the procedural complication rate was $9.1 \%(n=19 ; 95 \%$ CI, 5.6\%-13.9\%). These results are presented in Table 2.

\section{Predictors of 90-Day Favorable Outcome}

Center-adjusted analyses of predictors of favorable outcome are detailed in On-line Table 3. In backward stepwise multivariable regression analysis, age, admission NIHSS score, hypertension, time intervals between onset to imaging and imaging to groin puncture, prestroke $m R S \geq 1$, general anesthesia, and non-M1 MCA occlusion were associated with a decrease in the favorable outcome rate, whereas intravenous thrombolysis was associated with an increase
(Table 3). This prognosis model had good discrimination (median C-statistic, 0.827; range, $0.816-0.838$ across imputed datasets).

\section{Predictors of Successful and Complete Reperfusion}

Center-adjusted analyses of predictors of successful and excellent reperfusion outcome are detailed in On-line Tables 4 and 5. A higher ASPECTS (OR per 1-point increase, 1.25; 95\% CI, $1.08-$ 1.45 ) and no current smoking (OR, 0.36; 95\% CI, 0.18-0.72) were independently associated with successful reperfusion in backward stepwise multivariate regression analysis (Table 3). This prognosis model had good discrimination (median C-statistic, 0.681; range, 0.672-0.688 across imputed datasets).

For excellent reperfusion, we identified 5 independent predictors: older age, previous antiplatelet medications, lower blood glucose, site of occlusion (with a greater rate of excellent reperfusion for MCA M1 and vertebrobasilar occlusions), and Sofia $6 \mathrm{~F}$ catheters (Table 3). This prognosis model also had good discrimination (median C-statistic, 0.735; range, 0.725-0.741 across imputed datasets).

\section{Predictors of First-Pass Effect (mTICI 3 after 1 Pass)}

Center-adjusted analyses of predictors of FPE are detailed in Online Table 6. We identified 3 independent predictors: older age, site of occlusion (with a greater rate of excellent reperfusion after 1 pass for MCA M1 and verterbobasilar occlusions), and intravenous thrombolysis (Table 3 ). This prognosis model also showed good discrimination (median C-statistic, 0.795; range, 0.7930.795 across imputed datasets).

\section{DISCUSSION}

Our study demonstrated a very high efficacy of first-line contact aspiration using the Sofia catheter with $86.1 \%$ successful reperfusion (mTICI 2b, 2c/3) and 43\% favorable clinical outcome (mRS $<3)$. The FPE rate was high $(24.2 \%)$. Factors associated with higher recanalization rates were, notably, $\mathrm{M} 1$ and vertebrobasilar occlusions and the use of a $6 \mathrm{~F}$ catheter.

\section{Clinical Outcomes}

As previously described, ${ }^{1,13}$ outcome was significantly negatively influenced by age, stroke severity assessed by admission NIHSS score, prestroke $m R S \geq 1$, general anesthesia, ICA terminus and tandem occlusions, and late treatment timeframe. Most interesting, IVT in combination with mechanical thrombectomy is associated with favorable outcome. These predictive factors have already been previously demonstrated with the use of either stent retrievers or the ADAPT technique.

The influence of general anesthesia on clinical outcomes is currently under debate. ${ }^{14}$ In our study, general anesthesia was not the first-line option for thrombectomy procedures among most participating centers. This anesthesia regimen was proposed for selected severe cases, thus likely explaining the association between general anesthesia and poorer outcomes in our work.

Despite several studies demonstrating the association between IVT and favorable outcome in cases of stroke treated with thrombectomy, ${ }^{15}$ IVT influence is still discussed. Future randomized trials will probably bring answers. 
Table 2: Efficacy and safety outcomes in the overall population and population without rescue therapy $(n=296)^{a}$

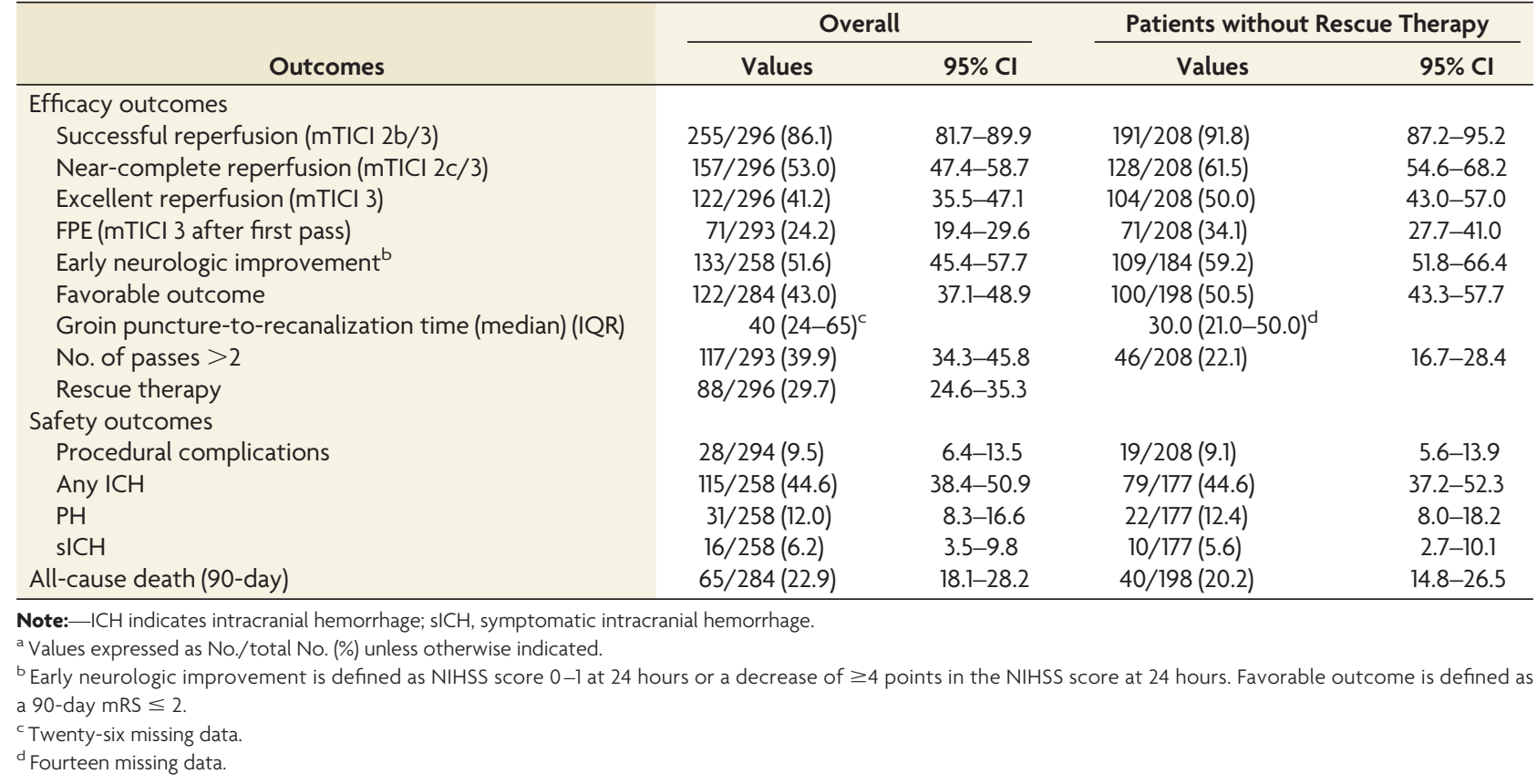

\section{Angiographic Outcome}

We present here the largest population treated with first-line ADAPT using Sofia catheters. Our final rate of favorable mTICI $(\geq 2 \mathrm{~b})$ appears similar to those previously published because authors noted favorable recanalization rates between $86.9 \%$ and $94.1 \%$ with the Sofia catheter. ${ }^{8-11}$ Gory et $\mathrm{al}^{16}$ reported $89 \%$ of mTICI $2 b / 3$ in a meta-analysis of the overall literature dealing with ADAPT using various kinds of DACs. In comparison, reperfusion rates among ADAPT major trails were $92 \%$ and $85.4 \%$, respectively, in the COMPASS and ASTER trials. Our results are consistent with these values.

Considering all types of mechanical thrombectomy approaches, these rates are among the best reported. For example, considering stent retrievers for most cases, the Highly Effective Reperfusion Evaluated in Multiple Endovascular Stroke (HERMES) trial meta-analysis revealed $71 \%$ successful reperfusion (mTICI $2 \mathrm{~b} / 3$ ). ${ }^{1}$ Among the stent retriever subgroups of both the COMPASS and ASTER trials, final successful recanalization rates were, respectively, $89 \%$ and $83.1 \%$. Of course, the maximalist strategy combining contact aspiration and stent retriever is currently presented as a promising technique. Previous studies reported a rate of $72.4 \%$ with the Aspiration-Retriever Technique for Stroke (ARTS) approach. ${ }^{17} \mathrm{New}$ approaches such as Proximal Balloon Occlusion Together with Direct Thrombus Aspiration during Stent Retriever Thrombectomy (PROTECT $\left.{ }^{\text {Plus }}\right)^{18}$ and Stent Retriever Assisted Vacuum-Locked Extraction (SAVE) techniques $^{19}$ seem to achieve very high revascularization levels. However, these findings can be balanced with the advantages of ADAPT as a first-line approach: ease of use, high effectiveness, and cost-effectiveness.

High-quality revascularization (mTICI $2 c / 3$ ) is now considered a strong prognostic factor. ${ }^{20} \mathrm{We}$ found that Sofia distal aspiration alone could achieve mTICI $2 c / 3$ and mTICI 3 results in $53 \%$ and $41.2 \%$. These rates are-as other indicators previously reported here-close to recently published data. ${ }^{5,21}$
In our study, rescue therapy using a stent retriever was required in $29.7 \%$. This was a low rate, enhancing the high-efficacy level of aspiration first-pass alone in our experience. In the comparable current literature, rescue therapy after the ADAPT technique was needed in 31\%-40\%., ${ }^{6,22-24}$ Lower rescue therapy rates have been published with stent retrievers. ${ }^{25}$ However, this has to be questioned because the stent retriever approaches are often already associated with an intermediate catheter able to perform combined aspiration and thus can be seen as a maximalist strategy. Nevertheless, the probable need of a complementary rescue technique seems low.

Most interesting, we also emphasized a higher excellent reperfusion rate (mTICI 3 ) with a Sofia 6 F than Sofia 5F. This strongly suggested a need for the largest diameter DAC possible to reach higher rates of reperfusion. This confirms previously published data. $^{24,26,27}$

Our complication rate is low and in accordance with earlier reports. Moreover, the complication rate included benign events such as vasospasm. In the ASTER trial, ${ }^{6}$ the first-line contact aspiration group had a $16.2 \%$ complication rate, and the first-line stent retriever ASTER group had a 15.9\% complication rate (nonsignificant difference). Blanc et $\mathrm{al}^{22}$ also reported a $13.3 \%$ rate.

\section{First-Pass Effect}

The FPE has recently been described as a strong prognostic factor of favorable outcome. Zaidat et $\mathrm{al}^{12}$ reported an FPE rate of $25.1 \%$ using stent retrievers exclusively. We report here an FPE rate of $24.2 \%$. M1 and vertebrobasilar occlusions were significant predictors of FPE. These topographies appear to particularly fit with the first-line ADAPT strategy using the Sofia catheter. Predictors of FPE were also older age and intravenous thrombolysis. Zaidat et al found that older age was also associated with the FPE but only in univariate analysis. Most interesting, we found that IVT was 1 remarkable factor associated with a higher prevalence of FPE. This has not been described to date, to our knowledge. In our study, the 
Table 3: Independent predictors of favorable outcome; successful, excellent reperfusion; and first-pass effect ${ }^{a}$

\begin{tabular}{|c|c|c|}
\hline & OR $(95 \% \mathrm{Cl})$ & $P$ Value ${ }^{b}$ \\
\hline \multicolumn{3}{|l|}{ Favorable outcome } \\
\hline Age & $0.72(0.58-0.91)^{b}$ & .005 \\
\hline Admission NIHSS score & $0.86(0.81-0.91)^{c}$ & $<.001$ \\
\hline Prestroke $\mathrm{mRS} \geq 1$ & $0.37(0.17-0.78)$ & .009 \\
\hline Intravenous rtPA & $2.61(1.42-4.78)$ & .002 \\
\hline Site of occlusion & & .026 \\
\hline M1 MCA & 1.00 (ref) & \\
\hline M2 MCA & $0.81(0.31-2.09)$ & .66 \\
\hline T-car & $0.65(0.30-1.41)$ & .27 \\
\hline Tandem & $0.49(0.21-1.14)$ & .098 \\
\hline Vertebrobasilar & $0.15(0.05-0.49)$ & .002 \\
\hline General anesthesia & $0.36(0.17-0.76)$ & .008 \\
\hline \multicolumn{3}{|l|}{ Procedural time (min) } \\
\hline Onset to imaging & $0.87(0.77-0.97)^{\mathrm{d}}$ & .017 \\
\hline Imaging to groin puncture & $0.67(0.52-0.90)^{d}$ & .007 \\
\hline \multicolumn{3}{|l|}{ Successful reperfusion } \\
\hline Current smoking & $0.36(0.18-0.72)$ & .004 \\
\hline ASPECTS & $1.25(1.08-1.45)^{c}$ & .003 \\
\hline \multicolumn{3}{|l|}{ Excellent reperfusion } \\
\hline Age & $1.26(1.04-1.54)$ & .018 \\
\hline Antiplatelet drugs & $2.31(1.24-4.32)$ & .009 \\
\hline Blood glucose (mmol/L) & $0.87(0.77-0.97)$ & .012 \\
\hline Site of occlusion & & $<.001$ \\
\hline M1 MCA & 1.00 (ref) & \\
\hline M2 MCA & $0.86(0.35-2.14)$ & .75 \\
\hline T-car & $0.38(0.18-0.79)$ & .010 \\
\hline Tandem & $0.38(0.16-0.91)$ & .030 \\
\hline Vertebrobasilar & $2.55(1.11-5.85)$ & .027 \\
\hline \multicolumn{3}{|l|}{ Sofia catheter } \\
\hline$>5 \mathrm{~F}$ & $0.38(0.17-0.83)$ & .015 \\
\hline$>6 \mathrm{~F}$ & 1.00 (ref) & \\
\hline \multicolumn{3}{|l|}{ First-pass effect } \\
\hline Age & 1.48 (1.16-1.90) & .002 \\
\hline Site of occlusion & & $<.001$ \\
\hline M1 MCA & 1.00 (ref) & \\
\hline M2 MCA & $0.75(0.32-1.75)$ & \\
\hline T-car & $0.11(0.03-0.38)$ & \\
\hline Tandem & $0.13(0.03-0.60)$ & \\
\hline Vertebrobasilar & $2.46(1.07-5.75)$ & \\
\hline Intravenous rtPA & 2.01 (1.09-3.71) & .024 \\
\hline
\end{tabular}

Note:-ref indicates reference; T-Car, occlusion of carotid terminus.

a Factors included in multivariate analysis of favorable outcome: age, hta, previous stroke or TIA, systolic BP, admission NIHSS score and ASPECTS, prestroke mRS $\geq 1$, intravenous rtPA, general anesthesia, delay in imaging-to-groin puncture, site of occlusion, and the Sofia catheter. Factors included in multivariate model of successful reperfusion: age, current smoking, admission ASPECTS, site of occlusion, intravenous rtPA, etiology, delay in onset to imaging. Factors included in multivariate analysis of excellent reperfusion: age, sex, current smoking, antiplatelet use, glycemia, admission ASPECTS, site of occlusion, intravenous rtPA, and the Sofia catheter. Factors included in multivariate analysis of first-pass effect: age, current smoking, previous stroke or TIA, antiplatelet use, glycemia, admission NIHSS score and ASPECTS, prestroke mRS $\geq 1$, site of occlusion. and intravenous rtPA.

${ }^{b}$ Per 10-year increase.

c Per 1-point increase.

${ }^{\mathrm{d}}$ Per 1-hour increase.

use of a balloon-guide catheter was not significantly associated with FPE, but this could be explained by the rare use of balloonguide catheters in our population.

Recent publications reported very high rates of FPE. ${ }^{18,19}$ Once again, considering our results and literature data, the ADAPT approach, especially with large-bore catheters, has to be considered a reliable front-line technique. In particular, M1 and basilar occlusions are very favorable targets, as demonstrated here.

\section{Limitations}

One limitation of this study is its retrospective nature, which also limits the possibility of harmonizing endovascular procedures among different operators. This can constitute a bias in perioperative data analysis. Nevertheless, this point makes our results very likely to be representative of daily practice in different thrombectomy centers. The absence of core lab analysis might also constitute a limitation. Other potential limitations include differences in study-entry criteria and patient characteristics among the centers that may be a source of bias.

\section{CONCLUSIONS}

The Sofia catheter is an efficient thrombectomy tool, with a good efficacy and safety profile. We demonstrated very high reperfusion rates (mTICI $2 \mathrm{~b} / 3$ and FPE) with a first-line thrombectomy strategy using Sofia catheters. Further trials comparing different catheters may be useful to appreciate the efficacy and safety differences between these devices.

Disclosures: Bertrand Lapergue-UNRELATED: Consultancy: Stryker; Grants/ Grants Pending: research grant from MicroVention for supporting the ETIS Network, research grant for the ASTER 2 trial from Stryker, MicroVention, Penumbra*; Payment for Lectures Including Service on Speakers Bureaus: Bristol-Myers Squibb, Servier, Boehringer Ingelheim. Xavier Barreau—RELATED: Grant: Bordeaux University Hospital Neuroradiology Unit.* *Money paid to the institution.

\section{REFERENCES}

1. Goyal M, Menon BK, Van Zwam WH, et al; HERMES collaborators. Endovascular thrombectomy after large-vessel ischaemic stroke: a meta-analysis of individual patient data from five randomised trials. Lancet 2016;387:1723-31 CrossRef Medline

2. Turk AS, Spiotta A, Frei D, et al. Initial clinical experience with the ADAPT technique: a direct aspiration first pass technique for stroke thrombectomy. J Neurointerv Surg 2014;6:231-37 CrossRef Medline

3. Turk AS, Frei D, Fiorella D, et al. ADAPT FAST study: a direct aspiration first pass technique for acute stroke thrombectomy. $\mathrm{J} \mathrm{Neu}$ rointerv Surg 2014;6:260-64 CrossRef Medline

4. Stapleton CJ, Leslie-Mazwi TM, Torok CM, et al. A direct aspiration first-pass technique vs stentriever thrombectomy in emergent large vessel intracranial occlusions. J Neurosurg 2018;128:567-74 CrossRef Medline

5. Turk AS 3rd, Siddiqui A, Fifi JT, et al. Aspiration thrombectomy versus stent retriever thrombectomy as first-line approach for large vessel occlusion (COMPASS): a multicentre, randomised, open label, blinded outcome, non-inferiority trial. Lancet 2019;393:9981008 CrossRef Medline

6. Lapergue B, Blanc R, Gory B, et al; ASTER Trial Investigators. Effect of endovascular contact aspiration vs stent retriever on revascularization in patients with acute ischemic stroke and large vessel occlusion: the ASTER randomized clinical trial. JAMA 2017;318: 443-52 CrossRef Medline

7. Humphries W, Hoit D, Doss VT, et al. Distal aspiration with retrievable stent assisted thrombectomy for the treatment of acute ischemic stroke. J Neurointerv Surg 2015;7:90-94 CrossRef Medline

8. Stampfl S, Kabbasch C, Muller M, et al. Initial experience with a new distal intermediate and aspiration catheter in the treatment of acute ischemic stroke: clinical safety and efficacy. J Neurointerv Surg 2016;8:714-18 CrossRef Medline

9. Möhlenbruch MA, Kabbasch C, Kowoll A, et al. Multicenter experience with the new SOFIA Plus catheter as a primary local aspiration catheter for acute stroke thrombectomy. J Neurointerv Surg 2017;9: 1223-27 CrossRef Medline

10. Wong JY, Do HM, Telischak NA, et al. Initial experience with SOFIA as an intermediate catheter in mechanical thrombectomy 
for acute ischemic stroke. J Neurointerv Surg 2017;9:1103-06 CrossRef Medline

11. Shallwani H, Shakir HJ, Rangel-Castilla L, et al. Safety and efficacy of the Sofia (6F) PLUS distal access reperfusion catheter in the endovascular treatment of acute ischemic stroke. Clin Neurosurg 2018;82: 312-21 CrossRef Medline

12. Zaidat OO, Castonguay AC, Linfante I, et al. First pass effect: a new measure for stroke thrombectomy devices. Stroke 2018;49:660-66 CrossRef Medline

13. Hungerford JP, Hyer M, Turk AS, et al. Impact of ASPECT scores and infarct distribution on outcomes among patients undergoing thrombectomy for acute ischemic stroke with the ADAPT technique. J Neurointerv Surg 2017;9:823-29 CrossRef Medline

14. Sørensen LH, Speiser L, Karabegovic S, et al. Safety and quality of endovascular therapy under general anesthesia and conscious sedation are comparable: results from the GOLIATH trial. J Neurointerv Surg 2019 Mar 29. [Epub ahead of print] CrossRef Medline

15. Gariel F, Lapergue B, Bourcier R, et al; ASTER Trial Investigators. Mechanical thrombectomy outcomes with or without intravenous thrombolysis. Stroke 2018;49:2383-90 CrossRef Medline

16. Gory B, Armoiry X, Sivan-Hoffmann R, et al. A direct aspiration first pass technique for acute stroke therapy: a systematic review and meta-analysis. Eur J Neurol 2018;25:284-92 CrossRef Medline

17. Malisch TW, Zaidat OO, Castonguay C. Clinical and angiographic outcomes with the combined local aspiration and retriever in the North American Solitaire Stent-Retriever Acute Stroke (NASA) Registry. Interv Neurol 2018;7:26-35 CrossRef Medline

18. Maegerlein C, Berndt M, Mönch S, et al. Further development of combined techniques using stent retrievers, aspiration catheters and BGC: the PROTECT ${ }^{\text {PLUS }}$ technique. Clin Neuroradiol 2018 Nov 9. [Epub ahead of print] CrossRef Medline

19. Maus V, Henkel S, Riabikin A, et al. The SAVE technique: large-scale experience for treatment of intracranial large vessel occlusions. Clin Neuroradiol 2018 Jul 19. [Epub ahead of print] CrossRef Medline
20. Dargazanli C, Fahed R, Blanc R, et al. Modified Thrombolysis in Cerebral Infarction 2c/Thrombolysis in Cerebral Infarction 3 reperfusion should be the aim of mechanical thrombectomy: insights from the ASTER trial (Contact Aspiration Versus Stent Retriever for Successful Revascularization). Stroke 2018;49:1189-96 CrossRef Medline

21. Kaschner MG, Rubbert C, Caspers J, et al. A retrospective singlecenter case series of direct aspiration thrombectomy as first-line approach in ischemic stroke and review of the literature. J Stroke Cerebrovasc Dis 2019;28:640-48 CrossRef Medline

22. Blanc R, Redjem H, Ciccio G, et al. Predictors of the aspiration component success of A Direct Aspiration First Pass Technique (ADAPT) for the endovascular treatment of stroke reperfusion strategy in anterior circulation acute stroke. Stroke 2017;48: 1588-93 CrossRef Medline

23. Schramm P, Navia P, Papa R, et al. ADAPT technique with ACE68 and ACE64 reperfusion catheters in ischemic stroke treatment: results from the PROMISE study. J Neurointerv Surg 2019;11:226-31 CrossRef Medline

24. Alawieh A, Chatterjee AR, Vargas J, et al. Lessons learned over more than $\mathbf{5 0 0}$ stroke thrombectomies using ADAPT with increasing aspiration catheter size. Neurosurgery 2018 Nov 10. [Epub ahead of print] CrossRef Medline

25. Tsang CO, Cheung IH, Lau KK, et al. Outcomes of stent retriever versus aspiration-first thrombectomy in ischemic stroke: a systematic review and meta-analysis. AJNR Am J Neuroradiol 2018;39: 2070-76 CrossRef Medline

26. Nikoubashman O, Nikoubashman A, Büsen M, et al. Necessary catheter diameters for mechanical thrombectomy with ADAPT. AJNR Am J Neuroradiol 2017;38:2277-81 CrossRef Medline

27. Delgado Almandoz JE, Kayan Y, Wallace AN, et al. Larger ACE 68 aspiration catheter increases first-pass efficacy of ADAPT technique. J Neurointerv Surg 2019;11:141-46 CrossRef Medline 